\title{
Random-start GnRH antagonist for emergency fertility preservation: a self-controlled trial
}

This article was published in the following Dove Press journal:

International Journal of Women's Health

12 February 2015

Number of times this article has been viewed

\author{
Miguel A Checa',2 \\ Mario Brassesco ${ }^{2}$ \\ Margalida Sastre ${ }^{1}$ \\ Manuel Gómez ${ }^{2}$ \\ Julio Herrero ${ }^{3}$ \\ Laura Marque ${ }^{3}$ \\ Arturo Brassesco ${ }^{2}$ \\ Juan José Espinós ${ }^{3}$ \\ 'Department of Obstetrics \\ and Gynecology, Parc de Salut \\ Mar, Universitat Autònoma de \\ Barcelona, ${ }^{2}$ Centro de Infertilidad \\ y Reproducción Humana (CIRH), \\ ${ }^{3}$ Centro de Reproducción Asistida \\ Sagrada Familia, Clínica Sagrada \\ Familia, Barcelona, Spain
}

\begin{abstract}
The aim of this study is to evaluate the feasibility and safety of random-start controlled ovarian hyperstimulation $(\mathrm{COH})$ for emergency fertility preservation, regardless of the phase of the menstrual cycle. A self-controlled pilot clinical trial (NCT01385332) was performed in an acute-care teaching hospital and in two private reproductive centers in Barcelona, Spain. Eleven egg donors participated in the study. Two random-start gonadotropin-releasing hormone $(\mathrm{GnRH})$ antagonist protocols were assessed in which ganirelix was initiated on either day 10 (protocol B) or on day 20 (protocol C) of the menstrual cycle and was continued until estradiol levels were below $60 \mathrm{pg} / \mathrm{dL}$. These protocols were compared with a standard protocol (protocol A). The main outcome of interest was the number of metaphase 2 oocytes retrieved. Results from this study show that the number of mature oocytes retrieved was comparable across the different protocols (14.3 \pm 4.6 in the standard protocol versus $13.0 \pm 9.1$ and $13.2 \pm 5.2$ in protocols $\mathrm{B}$ and $\mathrm{C}$, respectively; values expressed as mean \pm standard deviation). The mean number of days needed for a GnRH antagonist to lower estradiol levels, as well as the ongoing pregnancy rates, were also similar when protocols B (stimulation in follicular phase) and $\mathrm{C}$ (stimulation on luteal phase) were compared with protocol A (standard stimulation). GnRH antagonists can be effectively used for random-start controlled ovarian hyperstimulation with an ovarian response similar to that of standard protocols, and the antagonists appear suitable for emergency fertility preservation in cancer patients.
\end{abstract}

Keywords: controlled ovarian hyperstimulation, GnRH antagonists, emergency fertility preservation, cancer patients

\section{Introduction}

Reproductive-age women who are diagnosed with a malignant disease face the potential risk of losing their fertility because of chemotherapy or radiation therapy and, therefore, they risk losing the opportunity to have children. ${ }^{1}$ In 2009 , the American Cancer Society predicted more than 190,000 new cases of breast cancer in women. ${ }^{2}$ They estimated that roughly 18,600 of these women would be younger than 45 years of age. ${ }^{2}$ In a study of 82,699 incident cases of breast cancer diagnosed in Spain during the period from 1980-2004, breast cancer increased annually by $1.7 \%$ during that period for women younger than 45 years of age. ${ }^{3}$ Also, in a survey of 657 young women with a history of early-stage breast cancer, $57 \%$ recalled having substantial concerns at the time of diagnosis about becoming infertile with treatment. ${ }^{4}$

The best approach and treatment options for cancer patients who are concerned about preserving and managing their fertility are still matters of debate, albeit fertility preservation approaches should be considered as early as possible during treatment
Correspondence: Miguel A Checa Department of Obstetrics and Gynecology, Parc de Salut Mar, Passeig Marítim 25-29, E-08003 Barcelona, Spain Fax +34 932483254

Email macheca@hospitaldelmar.cat 
planning. ${ }^{5}$ For women whose cancer treatment cannot be delayed, there is a narrow window of opportunity for egg harvesting. ${ }^{6}$ Vitrification of oocytes is an efficient method for oocyte cryopreservation, although previous controlled ovarian stimulation is necessary. ${ }^{7}$ The conventional approach requires approximately 2 weeks of ovulation induction from the beginning of the menstrual cycle; this process could entail a delay by up to 6 weeks for starting cancer treatment, depending on the phase of the menstrual cycle during which the patient is referred. ${ }^{6}$

The use of gonadotropin-releasing hormone (GnRH) antagonists has emerged as a convenient strategy to reduce the duration of the treatment cycle. ${ }^{8-11}$ However, there is limited evidence regarding the feasibility of $\mathrm{GnRH}$ antagonist protocols to initiate controlled ovarian hyperstimulation $(\mathrm{COH})$ at a random date in the menstrual cycle. Random-start $\mathrm{COH}$ could be a valuable approach for emergency fertility preservation in cancer patients. Therefore, a self-controlled, exploratory pilot clinical trial was designed to assess whether the use of $\mathrm{GnRH}$ antagonists to initiate $\mathrm{COH}$ at a random day of the menstrual cycle would allow the retrieval of the same number of mature oocytes as a standard long protocol would.

\section{Materials and methods Design and study population}

This self-controlled clinical trial was conducted on egg donors recruited consecutively at an acute-care teaching hospital and at two private reproductive medicine centers in Barcelona, Spain between January 2011 and December 2011. The purpose of this study was to determine the efficacy and safety of two new protocols for $\mathrm{COH}$, defined as the total number of retrieved oocytes. Although this study was performed on egg donors, these new protocols are intended to be implemented on fertility preservation patients. All participants gave written informed consent for ovarian stimulation and egg donation. In addition, all recipients who received embryos obtained from these protocols also gave written informed consent. The study protocol was approved by the Ethics Committee for Clinical Research of Parc de Salut Mar, Barcelona, Spain. The study was registered with ClinicalTrials.gov on May 28, 2010 (NCT01385332).

Women enrolled in the study were between 18 and 32 years of age and had no previous history of chemotherapy, exposure to any gonadotoxic drugs (for example, methotrexate), a history of ovarian surgery, nor infertility. Other inclusion criteria comprehended a body mass index (BMI) between $12 \mathrm{~kg} / \mathrm{m}^{2}$ and $28 \mathrm{~kg} / \mathrm{m}^{2}$, and baseline follicle-stimulating hormone (FSH) levels below
$10 \mathrm{mIU} / \mathrm{mL}$. Patients included were negative for hepatitis $\mathrm{C}$ virus, hepatitis B virus, human immunodeficiency virus, and syphilis infection. In addition, women with polycystic ovarian syndrome and gonadotropin allergies were excluded from this study. Male factor, defined by the World Health Organization criteria, was also considered an exclusion criterion. $^{12}$

All participants were scheduled in the early follicular phase (days 3-5 of the menstrual cycle) for a baseline evaluation in which serum levels of FSH, estradiol, and luteinizing hormone (LH) were measured. An antral follicle count (AFC) was performed using transvaginal ultrasonography on days 1 or 2 of the menstrual cycle.

\section{Ovarian stimulation cycles}

All women underwent two $\mathrm{COH}$ cycles. First, the standard $\mathrm{COH}$ protocol was initiated during the early follicular phase (protocol A, control group). Then, following a selfcontrolled, open-label design, patients underwent a second cycle of $\mathrm{COH}$ either in the midfollicular phase by starting a GnRH antagonist on day 10 of the menstrual cycle (protocol B), or in the early luteal phase by starting a GnRH antagonist on day 20 of the menstrual cycle (protocol C). Prior to the first $\mathrm{COH}$ cycle, participants were randomly assigned to protocol $\mathrm{B}$ or $\mathrm{C}$ using a computer-generated table of random numbers.

\section{Protocol A (control group)}

Ovarian stimulation was initiated on day 2 of the menstrual cycle with $225 \mathrm{IU} /$ day of recombinant FSH $(\mathrm{rFSH})$ (Puregon ${ }^{\circledR}$; Merck and Co, Inc., Whitehouse Station, NJ, USA) for 5 days. The dose of rFSH was adjusted for each patient according to the follicular growth detected by ultrasonography after the 5th day of rFSH administration. A GnRH antagonist (ganirelix; Orgalutran $^{\circledR}$; Merck and Co, Inc.) at a dose of $0.25 \mathrm{mg} / \mathrm{dL}$ per day was administered from day 6 of rFSH onwards to prevent LH surge. When at least three follicles at least $17 \mathrm{~mm}$ in diameter were observed by ultrasonography, a GnRH agonist (Procrin ${ }^{\circledR}$; Abbott Laboratories, Abbott Park, IL, USA) at a dose of $0.2 \mathrm{~mL} / \mathrm{IU}$ was given as a trigger. Oocytes were retrieved transvaginally 36 hours later and fertilized either by conventional in vitro fertilization (IVF) or by means of intracytoplasmic sperm injection (ICSI).

\section{Protocols $B$ and $C$ (intervention)}

In protocols $\mathrm{B}$ and $\mathrm{C}$, ganirelix treatment was initiated on day 10 (protocol B, midfollicular phase) or on day 20 (protocol C, early luteal phase) of the menstrual cycle at 
a dose of $0.25 \mathrm{mg} / \mathrm{dL}$ per day. Ganirelix treatment was given until serum estradiol levels were below $60 \mathrm{pg} / \mathrm{dL}$. Then, the standard protocol was started with $225 \mathrm{IU} /$ day of $\mathrm{rFSH}$ (Puregon $^{\circledR}$; Merck and Co, Inc.). As in protocol A, the dose of rFSH was adjusted depending on the individual ovarian response after the 5th day of treatment. A fixed dose of ganirelix, $0.25 \mathrm{mg} / \mathrm{dL}$ per day, was administered from day 6 of rFSH treatment onwards to prevent premature LH surges. When at least three follicles had reached at least $17 \mathrm{~mm}$ in diameter, $0.2 \mathrm{~mL} / \mathrm{IU}$ of the GnRH agonist Procrin ${ }^{\circledR}$ (Abbott Laboratories) was administered to induce ovulation. Oocytes were retrieved transvaginally 36 hours later and fertilized either by conventional IVF or ICSI. The details of protocols B and $\mathrm{C}$ are shown in Figure 1.

\section{Priming/transfer protocol}

Recipients of donor eggs were downregulated with $3.75 \mathrm{mg}$ of a GnRH agonist, Decapeptyl ${ }^{\circledR}$ (Ipsen Pharma, Paris, France), and endometrial priming was induced by a transdermal patch,

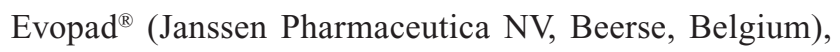
which released $75 \mu \mathrm{g}$ /day of estradiol. Natural micronized progesterone (Utrogestan ${ }^{\circledR}$; SEID S.A., Barcelona, Spain) at a dose of $800 \mathrm{mg} /$ day was used as luteal supplementation. A single embryo was transferred on day 3 .

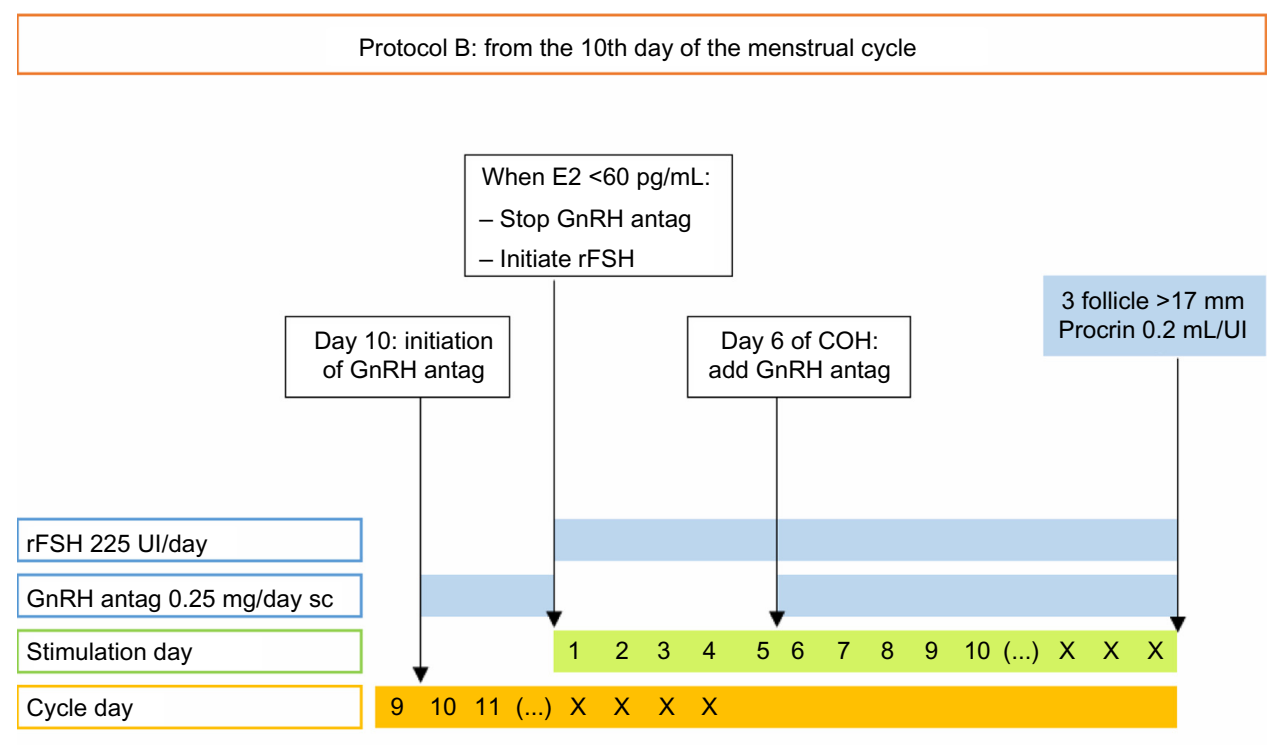

Protocol C: from the 20th day of the menstrual cycle

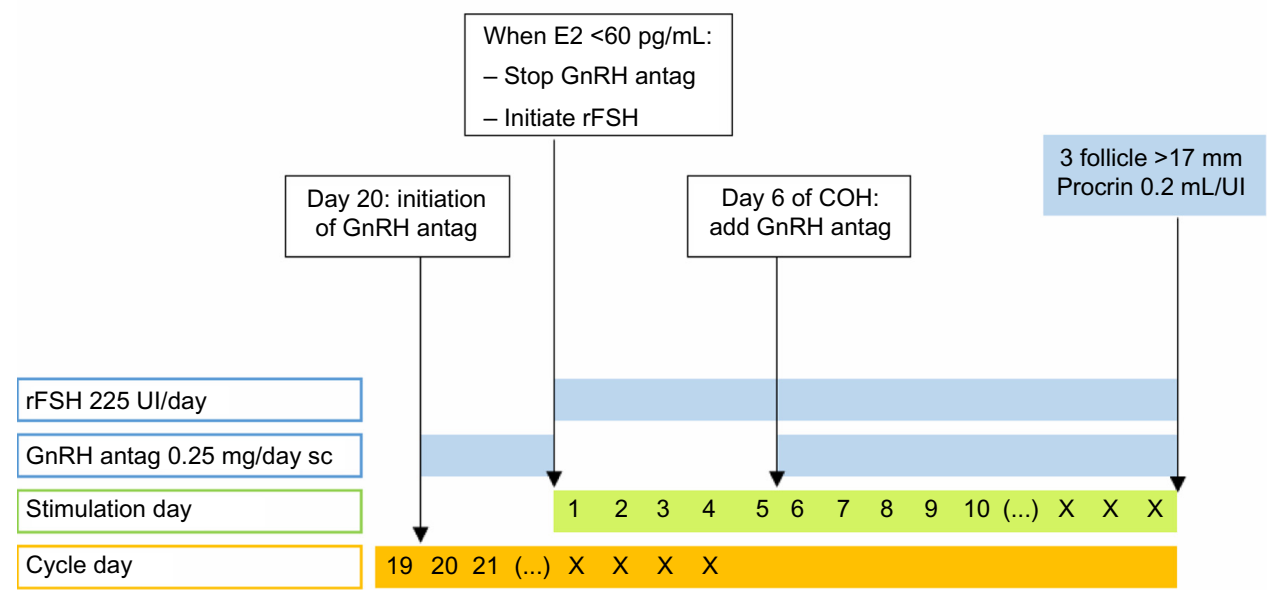

Figure I Random-start GnRH antagonist protocols in the midfollicular phase (protocol B) versus the early luteal phase (protocol C).

Abbreviations: E2, estradiol; GnRH, gonadotropin-releasing hormone; antag, antagonist; sc, subcutaneous; rFSH, recombinant follicle-stimulating hormone; $\mathrm{COH}$, controlled ovarian hyperstimulation. 


\section{Outcome measures}

The number of mature oocytes, defined as the number of metaphase 2 oocytes retrieved, was the main outcome of interest. Participants were followed for 1-3 hours after follicular aspiration. Fertilized embryos were classified on day 3 in accordance with the Spanish Association of Reproductive Biology (ASEBIR) embryo assessment criteria (ASEBIR, 2008). Egg recipients were followed until the 12 th week of pregnancy.

\section{Analysis}

Sample size calculation was not considered because of the exploratory design of this pilot study. It was estimated that five egg donors per group (with each participant being her own control) would be sufficient to assess the efficacy and safety of the two new protocols for $\mathrm{COH}$. Continuous variables are expressed as the mean values \pm standard deviation (SD), and categorical variables as frequencies and percentages. Differences in quantitative variables between the control group and the corresponding intervention groups (protocol A versus protocol B; protocol A versus protocol C) were analyzed with the Wilcoxon signed-rank test for paired samples, and differences in categorical variables with the McNemar's test. Statistical significance was set at a $P$-value $<0.05$. Analysis was performed with the Statistical Package for the Social Sciences (IBM Corporation, Armonk, NY, USA), version 15.0 for Windows.

\section{Results}

A total of 15 eligible egg donors were invited to participate in the study, although one woman refused to sign the informed consent. Therefore, 14 patients participated in this self-controlled clinical study. In the early luteal arm, two women were excluded. (For one participant, $\mathrm{COH}$ with the standard protocol was cancelled, and the other participant abandoned the study prior to the second stimulation for personal reasons). In the midfollicular arm, one participant abandoned the study for personal reasons. Therefore, six participants were included in the analysis of midfollicular GnRH intervention (protocol B) and five in the analysis of the early luteal GnRH intervention (protocol C). The flow chart of the study population is shown in Figure 2.

Baseline characteristics of the participants are shown in Table 1 . There were no statistically significant differences in terms of age, BMI, and pretreatment serum levels of FSH or AFC across the study groups.

The numbers of mature oocytes retrieved were similar for both protocols $\mathrm{B}$ and $\mathrm{C}$ and the standard protocol (mean $\pm \mathrm{SD}$,

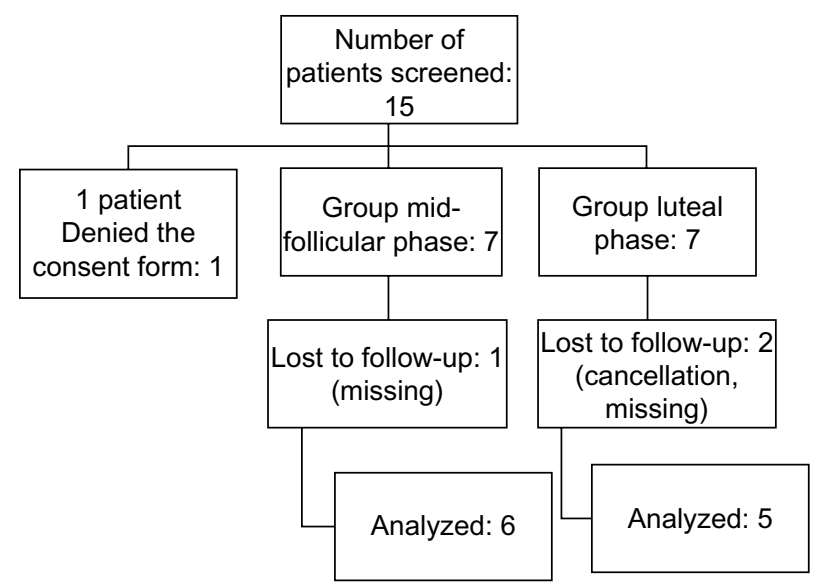

Figure 2 Flow chart of the study population.

Note: Each number represents the number of people in the category.

$14.3 \pm 4.6$ in the standard protocol versus $13.0 \pm 9.1$ and $13.2 \pm 5.2$ in protocols $\mathrm{B}$ and $\mathrm{C}$, respectively, as shown in Table 2). In addition, no differences were observed in terms of the fertilization rate, the percentage of top-quality embryos achieved, or the mean number of frozen embryos when the standard protocol was compared with either the midfollicular phase protocol or the early luteal phase protocol, as shown in Table 2.

The mean \pm SD number of days of GnRH antagonist treatment needed to decrease serum estradiol levels below $60 \mathrm{pg} / \mathrm{dL}$ was $2.8 \pm 0.8$ days in protocol B and 3.6 \pm 1.6 days in protocol $\mathrm{C}$ (Table 2). In addition, patients were on ganirelix during more days for protocols $\mathrm{B}$ and $\mathrm{C}$ than for the standard protocol (Protocol B: $5.8 \pm 1.9$ versus $7.8 \pm 3.3$ in the midfollicular arm; and Protocol C: $6.5 \pm 0.6$ versus $8.2 \pm 3.5$ in the early luteal arm; although the differences were not statistically significant). The mean numbers of days of stimulation were also similar for both GnRH antagonist protocols B and $\mathrm{C}$ and for the standard $\mathrm{COH}$ protocol (Table 2). Additionally, the ongoing pregnancy rates for egg recipients were also similar in both arms when comparing protocols $\mathrm{B}$ or $\mathrm{C}$ with

Table I Baseline characteristics of the participants included in the study

\begin{tabular}{llll}
\hline Variable & $\begin{array}{l}\text { Total } \\
\text { women } \\
(\mathbf{n}=\mathrm{II})\end{array}$ & $\begin{array}{l}\text { Midfollicular } \\
\text { phase } \\
(\mathbf{n}=\mathbf{6})\end{array}$ & $\begin{array}{l}\text { Early luteal } \\
\text { phase } \\
(\mathbf{n}=5)\end{array}$ \\
\hline Age, years & $25.8 \pm 3.7$ & $25.6 \pm 3.8$ & $26.0 \pm 4.0$ \\
BMI, $\mathrm{kg} / \mathrm{m}^{2}$ & $23.4 \pm 3.5$ & $22.6 \pm 2.6$ & $24.1 \pm 4.1$ \\
Serum FSH, $\mathrm{mIU} / \mathrm{mL}$ & $6.9 \pm 1.3$ & $7.1 \pm 1.5$ & $6.8 \pm 1.3$ \\
AFC & $11.5 \pm 2.6$ & $12.8 \pm 3.6$ & $10.6 \pm 1.4$ \\
\hline
\end{tabular}

Note: Values are expressed as the mean \pm standard deviation.

Abbreviations: $n$, number; BMI, body mass index; $F S H$, follicle-stimulating hormone; AFC, antral follicle count. 
Table 2 Within-group comparisons for both $\mathrm{COH}$ protocols with ganirelix

\begin{tabular}{|c|c|c|c|c|c|c|}
\hline Variables & $\begin{array}{l}\text { Standard } \\
\text { COH } \\
\text { (protocol A) }\end{array}$ & $\begin{array}{l}\text { Midfollicular } \\
\text { phase } \\
\text { (protocol B) }\end{array}$ & $P$-value & $\begin{array}{l}\text { Standard } \\
\text { COH } \\
\text { (protocol A) }\end{array}$ & $\begin{array}{l}\text { Early luteal } \\
\text { phase } \\
\text { (protocol C) }\end{array}$ & $P$-value \\
\hline Total women & 6 & 6 & & 5 & 5 & \\
\hline Total number of oocytes & $24.8 \pm 13.0$ & $19.2 \pm 11.1$ & 0.141 & $17.0 \pm 7.9$ & $18.4 \pm 10.6$ & 0.892 \\
\hline Mature oocytes (metaphase 2) & $16.2 \pm 4.1$ & $13.0 \pm 9.1$ & 0.225 & $12.4 \pm 5.2$ & $13.2 \pm 5.2$ & 0.786 \\
\hline Immature oocytes & $8.6 \pm 10.6$ & $6.2 \pm 8.5$ & 0.066 & $4.6 \pm 4.6$ & $5.2 \pm 5.5$ & 0.686 \\
\hline Fertilization rate & $76.3 \%$ & $75.9 \%$ & 0.268 & $75.8 \%$ & $78.1 \%$ & 0.573 \\
\hline Top-quality embryos & $45.4 \%$ & $46.2 \%$ & 0.451 & $47.1 \%$ & $46.5 \%$ & 0.853 \\
\hline Frozen embryos & $4.3 \pm 2.8$ & $4.1 \pm 2.1$ & 0.278 & $3.9 \pm 1.9$ & $4.6 \pm 2.3$ & 0.342 \\
\hline Total $\mathrm{rFSH}$ dose, $\mathrm{mlU} / \mathrm{mL}$ & $\mathrm{I}, 680 \pm 445$ & $1,595 \pm 300$ & 0.500 & $1,966.7 \pm 559$ & $1,837.5 \pm 580$ & 0.753 \\
\hline Days on ganirelix & $5.8 \pm 1.9$ & $7.8 \pm 3.3$ & 0.180 & $6.5 \pm 0.6$ & $8.2 \pm 3.5$ & 0.581 \\
\hline Days of stimulation & $10.4 \pm 1.5$ & $9.8 \pm 0.8$ & 0.257 & $12.2 \pm 1.9$ & $10.6 \pm 2.1$ & 0.221 \\
\hline Clinical pregnancy rate, n (\%) & $3(50)$ & $6(100)$ & & $2(40)$ & $3(60)$ & \\
\hline Ongoing pregnancy rate, n (\%) & $3(50)$ & $5(83.3)$ & & $2(40)$ & $2(40)$ & \\
\hline
\end{tabular}

Note: Data are expressed as the mean \pm standard deviation unless otherwise stated.

Abbreviations: $\mathrm{COH}$, controlled ovarian hyperstimulation; $r F S H$, recombinant follicle-stimulating hormone; $n$, number.

the standard protocol $(83.3 \%$ versus $50 \%$ in the midfollicular arm, and $40 \%$ versus $40 \%$ in the early luteal arm, respectively, as shown in Table 2).

No cases of ovarian hyperstimulation syndrome (OHSS) were recorded.

\section{Discussion}

The results of this exploratory pilot study, conducted for ethical reasons in egg donors rather than in cancer patients, show that $\mathrm{COH}$ can be initiated at a random date in the menstrual cycle when GnRH antagonists are used to achieve estradiol suppression. The number of mature oocytes retrieved when ganirelix was used in both midfollicular and early luteal phases was similar to that retrieved with the standard $\mathrm{COH}$ protocol, independent of whether ganirelix was given on day 10 or day 20 of the menstrual cycle.

The available evidence regarding the different emergency fertility preservation strategies for $\mathrm{COH}$ and their effectiveness is sparse. In this regard, various case reports and series of short cases on random-start $\mathrm{COH}$ have been published with encouraging results. ${ }^{6,13,14}$ In the study by Sönmezer et al, ${ }^{6}$ random-start $\mathrm{COH}$ on three patients diagnosed with breast cancer commenced immediately on menstrual cycle days 11,14 , or 17 with the use of letrozole to suppress estradiol levels; between nine and 17 oocytes were harvested from each patient. Meanwhile, Ozkaya et $\mathrm{al}^{13}$ initiated randomstart $\mathrm{COH}$ on a patient, who was diagnosed with Hodgkin lymphoma, on day 11 of her menstrual cycle with the use of the $\mathrm{GnRH}$ antagonist ganirelix from day 1 of $\mathrm{COH}$; from this patient, 17 mature oocytes were obtained. Similarly, in the study by Nayak and Wakim, ${ }^{14}$ a random-start GnRH antagonist cycle with cetrorelix from day 1 of $\mathrm{COH}$ was used on four patients, two of whom were on the 10th day of their menstrual cycles, one of whom was on the 17 th day of her menstrual cycle, and one whose day of her menstrual cycle was unknown; between six and 30 mature oocytes were harvested from each of these patients. In addition, Kuang et $\mathrm{al}^{15}$ described how luteal phase ovarian stimulation with human menopausal gonadotropin and letrozole after spontaneous ovulation was also a feasible option for producing high-quality oocytes and embryos (11.2 \pm 7.2 oocytes were harvested from the human menopausal gonadotropin group, and $4.8 \pm 4.1$ oocytes from the letrozole group); optimal pregnancy outcomes after embryo transfer were achieved. ${ }^{15}$ However, because of the nature of these study designs, no control group was used to compare the results; hence, the applicability of these results must be interpreted in this context.

Findings from our trial indicate that the duration of an ovarian stimulation cycle for egg retrieval can be shorter compared to that of the standard protocols of $\mathrm{COH}$. According to the standard protocols, if a reproductive-age cancer patient is referred for emergency fertility preservation on day 10 of her menstrual cycle and has regular menses, clinicians should wait until day 2 of the following menstrual cycle to initiate $\mathrm{COH}$ (an approximately 20-day period). However, when the proposed GnRH antagonist ganirelix protocol was followed, ovarian stimulation was initiated $2.8 \pm 0.8$ days after the midfollicular phase started and was finished in $9.8 \pm 0.8$ days. Similar results were obtained when the GnRH antagonist was used during the early luteal phase; these results allowed clinicians to initiate $\mathrm{COH} 3.6 \pm 1.6$ days after the phase started and proceed to oocyte retrieval after 10.6 \pm 2.1 days. Surprisingly, the implementation of this protocol entailed 
shorter time intervals than those observed in fertility preservation programs through oocyte cryopreservation via vitrification. ${ }^{16,17}$

Interestingly, results from this trial did not show differences in terms of the mean numbers of immature oocytes retrieved among participants who underwent either the midfollicular phase or the early luteal phase protocols when compared to the mean number of oocytes obtained during the standard $\mathrm{COH}$ protocol ( $6.2 \pm 8.5$ versus $8.6 \pm 10.6$ in the midfollicular arm, and $5.2 \pm 5.5$ versus $4.6 \pm 4.6$ in the early luteal arm, respectively). The study showed no differences in either oocyte quality and maturation or pregnancy rates; this finding suggests that the fertility potential of the oocytes was not altered. Ongoing pregnancy rates were similar for the early luteal phase protocol and for the standard protocol ( $40 \%$ versus $40 \%$, respectively). However, in the midfollicular arm, higher rates were observed with the midfollicular protocol compared to those with the standard protocol (83.3\% versus 50\%). However, these could be random differences due to the small sample size. Nevertheless, in a study by Budak et $\mathrm{al}^{18}$ regarding the experience in oocyte donation cycles, the results showed an improvement in ongoing pregnancy rates, which increased from $31 \%$ to $44.3 \%$ over a period of 10 years. ${ }^{18}$ The latter results are consistent with the data obtained in our study.

The higher ovarian response obtained with the midfollicular phase protocol than with the early luteal phase protocol could be explained by differences in the baseline AFC between the two groups. Interestingly, differences in terms of AFC in the baseline characteristics comparison were not statistically significant; however, it would be reasonable to consider that the small sample size could interfere with the accuracy of these results. AFC is a well known marker of ovarian response for controlled ovarian stimulation in conventional IVF and ICSI. ${ }^{19,20}$ Finally, an additional advantage of applying a GnRH antagonist cycle with a $\mathrm{GnRH}$ agonist trigger is to prevent OHSS, whose incidence is reduced significantly by this type of treatment; thereby, this approach constitutes a safe strategy for egg donors. ${ }^{10}$ None of our patients had OHSS, despite the large number of oocytes retrieved.

The main limitations of this study include the exploratory and pilot nature of the trial and the small sample size. However, this is the first trial to assess the efficacy and safety of two different random-start GnRH antagonist protocols in two different phases of the menstrual cycle. Results from this trial show that GnRH antagonists are an effective and safe strategy to initiate $\mathrm{COH}$ at a random date to achieve an ovarian response similar to that obtained with the standard $\mathrm{COH}$ protocol. This new approach of emergency fertility preservation overcomes the inconveniences of waiting for the menstrual period to initiate and responds to the urgent treatment needs of cancer patients. However, larger randomized controlled studies are necessary to confirm these findings.

\section{Acknowledgments}

We thank Marta Pulido, MD, for editing the manuscript and for editorial assistance.

\section{Disclosure}

The authors report no conflicts of interest in this work.

\section{References}

1. Martínez F, Devesa M, Coroleu B, et al. Cancer and fertility preservation: Barcelona consensus meeting. Gynecol Endocrinol. 2013;29(4):285-291.

2. American Cancer Society. Breast Cancer Facts and Figures 2009-2010. Atlanta, GA: American Cancer Society, Inc.; 2009. Available from: http://www.cancer.org/acs/groups/content/@nho/documents/document/ f861009final90809pdf.pdft. Accessed October 10, 2013.

3. Pollán M, Michelena MJ, Ardanaz E, Izquierdo A, Sánchez-Pérez MJ, Torrella A; Breast Cancer Working Group. Breast cancer incidence in Spain before, during and after the implementation of screening programmes. Ann Oncol. 2010;21 Suppl 3:iii97-iii102.

4. Partridge AH, Gelber S, Peppercorn J, et al. Web-based survey of fertility issues in young women with breast cancer. J Clin Oncol. 2004;22(20):4174-4183.

5. Loren AW, Mangu PB, Beck LN, et al; American Society of Clinical Oncology. Fertility preservation for patients with cancer: American Society of Clinical Oncology clinical practice guideline update. J Clin Oncol. 2013;31(19):2500-2510.

6. Sönmezer M, Türkçüoğlu I, Coşkun U, Oktay K. Random-start controlled ovarian hyperstimulation for emergency fertility preservation in letrozole cycles. Fertil Steril. 2011;95(6):2125. e9-e11.

7. Kuwayama M, Vajta G, Kato O, Leibo SP. Highly efficient vitrification method for cryopreservation of human oocytes. Reprod Biomed Online. 2005;11(3):300-308.

8. Albano C, Felberbaum RE, Smitz J, et al. Ovarian stimulation with HMG: results of a prospective randomized phase III European study comparing the luteinizing hormone-releasing hormone (LHRH)antagonist cetrorelix and the LHRH-agonist buserelin. European Cetrorelix Study Group. Hum Reprod. 2000;15(3):526-531.

9. Al-Inany HG, Abou-Setta AM, Aboulghar M. Gonadotrophin-releasing hormone antagonists for assisted conception: a Cochrane review. Reprod Biomed Online. 2007;14(5):640-649.

10. Galindo A, Bodri D, Guillén JJ, Colodrón M, Vernaeve V, Coll O. Triggering with $\mathrm{HCG}$ or $\mathrm{GnRH}$ agonist in $\mathrm{GnRH}$ antagonist treated oocyte donation cycles: a randomised clinical trial. Gynecol Endocrinol. 2009;25(1):60-66.

11. Fatemi HM, Blockeel C, Devroey P. Ovarian stimulation: today and tomorrow. Curr Pharm Biotechnol. 2012;13(3):392-397.

12. Cooper TG, Noonan E, von Eckardsteins S, et al. World Health Organization reference values for human semen characteristics. Hum Reprod Update. 2010;16(3):231-245.

13. Ozkaya E, San Roman G, Oktay K. Luteal phase GnRHa trigger in random start fertility preservation cycles. J Assist Reprod Genet. 2012;29(6):503-505.

14. Nayak SR, Wakim AN. Random-start gonadotropin-releasing hormone $(\mathrm{GnRH})$ antagonist-treated cycles with GnRH agonist trigger for fertility preservation. Fertil Steril. 2011;96(1):e51-e54. 
15. Kuang Y, Hong Q, Chen Q, et al. Luteal-phase ovarian stimulation is feasible for producing competent oocytes in women undergoing in vitro fertilization/intracytoplasmic sperm injection treatment, with optimal pregnancy outcomes in frozen-thawed embryo transfer cycles. Fertil Steril. 2014;101(1):105-111.

16. Cobo A, Kuwayama M, Pérez S, Ruiz A, Pellicer A, Remohí J. Comparison of concomitant outcome achieved with fresh and cryopreserved donor oocytes vitrified by the Cryotop method. Fertil Steril. 2008;89(6):1657-1664.

17. Checa Vizcaíno MA, Corchado AR, Cuadri ME, Comadran MG, Brassesco M, Carreras R. The effects of letrozole on ovarian stimulation for fertility preservation in cancer-affected women. Reprod Biomed Online. 2012;24(6):606-610.
18. Budak E, Garrido N, Soares SR, et al. Improvements achieved in an oocyte donation program over a 10-year period: sequential increase in implantation and pregnancy rates and decrease in high-order multiple pregnancies. Fertil Steril. 2007;88(2):342-349.

19. Melo MA, Garrido N, Alvarez C, et al. Antral follicle count (AFC) can be used in the prediction of ovarian response but cannot predict the oocyte/embryo quality or the in vitro fertilization outcome in an egg donation program. Fertil Steril. 2009;91(1):148-156.

20. Checa MA, Prat M, Carreras R. Antral follicle count as a predictor of hyperresponse in controlled ovarian hyperstimulation/intrauterine insemination in unexplained sterility. Fertil Steril. 2010;94(3): $1105-1107$.
International Journal of Women's Health

\section{Publish your work in this journal}

The International Journal of Women's Health is an international, peerreviewed open-access journal publishing original research, reports, editorials, reviews and commentaries on all aspects of women's healthcare including gynecology, obstetrics, and breast cancer. The manuscript management system is completely online and includes

\section{Dovepress}

a very quick and fair peer-review system, which is all easy to use. Visit http://www.dovepress.com/testimonials.php to read real quotes from published authors.

\footnotetext{
Submit your manuscript here: http://www.dovepress.com/international-journal-of-womens-health-journal
} 